\title{
The role of the input on the development of the LC bias: A crosslinguistic comparison
}

\author{
Nayeli Gonzalez-Gomez ${ }^{\mathrm{a}, \mathrm{b}, *}$, Akiko Hayashi ${ }^{\mathrm{c}}$, Sho Tsuji ${ }^{\mathrm{d}, \mathrm{e}}$, Reiko Mazuka ${ }^{\mathrm{f}, \mathrm{g}}$, Thierry Nazzi ${ }^{\mathrm{a}, \mathrm{h}}$ \\ a Université Paris Descartes, Sorbonne Paris Cité, Paris, France \\ ${ }^{\mathrm{b}}$ Department of Psychology, Oxford Brookes University, UK \\ ${ }^{\mathrm{c}}$ Center for Research and Support of Educational Practice, Tokyo Gakugei University, Japan \\ ${ }^{\mathrm{d}}$ Radboud University Nijmegen, The Netherlands \\ e IMPRS for Language Sciences, The Netherlands \\ ${ }^{\mathrm{f}}$ Laboratory for Language Development, RIKEN Brain Sciences Institute, Japan \\ ${ }^{\mathrm{g}}$ Duke University, USA \\ ${ }^{\mathrm{h}}$ CNRS, Laboratoire Psychologie de la Perception, Paris, France
}

\section{A R T I C L E I N F O}

\section{Article history:}

Received 1 August 2013

Revised 11 April 2014

Accepted 12 April 2014

\section{Keywords:}

Infants

Speech perception

Phonological acquisition

Phonotactics

Perception/production link

Labial-Coronal bias

\begin{abstract}
A B S T R A C T
Previous studies have described the existence of a phonotactic bias called the Labial-Coronal (LC) bias, corresponding to a tendency to produce more words beginning with a labial consonant followed by a coronal consonant (i.e. "bat") than the opposite CL pattern (i.e. "tap"). This bias has initially been interpreted in terms of articulatory constraints of the human speech production system. However, more recently, it has been suggested that this presumably language-general LC bias in production might be accompanied by LC and CL biases in perception, acquired in infancy on the basis of the properties of the linguistic input. The present study investigates the origins of these perceptual biases, testing infants learning Japanese, a language that has been claimed to possess more CL than LC sequences, and comparing them with infants learning French, a language showing a clear LC bias in its lexicon. First, a corpus analysis of Japanese IDS and ADS revealed the existence of an overall LC bias, except for plosive sequences in ADS, which show a CL bias across counts. Second, speech preference experiments showed a perceptual preference for CL over LC plosive sequences (all recorded by a Japanese speaker) in 13- but not in 7- and 10-month-old Japanese-learning infants (Experiment 1), while revealing the emergence of an LC preference between 7 and 10 months in French-learning infants, using the exact same stimuli. These crosslinguistic behavioral differences, obtained with the same stimuli, thus reflect differences in processing in two populations of infants, which can be linked to differences in the properties of the lexicons of their respective native languages. These findings establish that the emergence of a $\mathrm{CL} / \mathrm{LC}$ bias is related to exposure to a linguistic input.
\end{abstract}

(c) 2014 Elsevier B.V. All rights reserved.

\footnotetext{
* Corresponding author. Address: Oxford Brookes University, Department of Psychology, Gipsy Lane, Oxford OX3 OBP, UK. Tel.: +44 186548 3719.
}

E-mail address: nayeli.gonzalez.gomez@gmail.com (N. Gonzalez-Gomez).

\section{Introduction}

Studies focusing on the analysis of the lexicons of various natural languages have revealed the existence of different phonotactic tendencies which occur consistently and crosslinguistically. The study of the origins of these consistent patterns across languages is a recurrent theme in the 
study of human cognition, as they are taken to reflect properties and constraints of the mechanisms underlying language processing and acquisition. For example, at the syllabic level, languages privilege open syllables (Consonant-Vowel, e.g. / $\mathrm{ma} /$ ) over closed syllables (Vowel-Consonant, e.g. /am/; Kawasaki-Fukumori, 1992; Rousset, 2003) and they privilege Consonant-Vowel sequences sharing the same place of articulation (e,g. /de/ or / ko/ rather than /ke/ or /do/; MacNeilage \& Davis, 2000a). At the intersyllabic level, languages have been shown to favor syllable sequences where consonants are articulatorily different (e.g. /baga/) over reduplications (e.g. /baba/; RochetCapellan \& Schwartz, 2005). In addition, among these variegated forms, sequences starting with a labial consonant followed by a coronal consonant (e.g. /bat/) are privileged over the opposite pattern (e.g. /tap/; MacNeilage, Davis, Kinney, \& Matyear, 1999; MacNeilage \& Davis, 2000b; Vallée, Rousset, \& Boë, 2001; Gonzalez-Gomez \& Nazzi, 2012a). This tendency is known as the Labial-Coronal (LC) bias. The present study will explore one factor that determines the emergence of the LC bias during development, frequency in the input, therefore focusing on the role of perceptual constraints by comparing infants learning either French, a language with an LC bias in its lexicon, or Japanese, a language that had been proposed to have a lexicon that does not have a clear LC bias (an issue we come back to in more details in the following). This kind of study is crucial to better understand influences of the perceptual and production systems as they support a robust phonotactic phenomenon observed cross linguistically.

The Labial-Coronal bias was first found in early production studies. During the 50-word-stage (12-18 months), infants tend to produce more Labial-Coronal (LC) than Coronal-Labial (CL) structures (Ingram, 1974; Locke, 1983; MacNeilage \& Davis, 2000a; MacNeilage et al., 1999). This tendency was found in 9 out of the 10 English-learning infants tested by MacNeilage et al. (1999) and in 21 out of the 22 infants learning English, German, Dutch, French or Czech (MacNeilage \& Davis, 2000a). Furthermore, the LC bias has not only been found in infant production studies but also in two typological studies examining 17 different languages (English, Estonian, French, German, Hebrew, Maori, Quechua and Spanish: MacNeilage et al., 1999; Afar, Finnish, French, Kannada, Kwalkw'ala, Navaho, Ngizim, Quechua, Sora and Yup'ik: Vallée et al., 2001).

The first interpretations for the origins of this bias were articulatory. One interpretation was offered within the frame-content theory (MacNeilage \& Davis, 1990; MacNeilage, 1998; Davis \& MacNeilage, 2004). It was proposed that infants tend to begin an utterance with a lip closure and then follow with a tongue contact closure (MacNeilage \& Davis, 2000a; MacNeilage, Davis, Kinney, \& Matyear, 2000). Since Labial-Vowel sequences are proposed to be pure frames (sequences that are produced by lip closure enabled by rhythmic mandibular oscillations without independent movement of the tongue within the sequence), while Coronal-Vowel sequences are fronted frames (sequences based on rhythmic mandibular oscillations with pre-positioning of the tongue at the alveolar ridge; no independent movement of the tongue from this pre-set position within the CV sequence), infants would tend to start with a labial consonant and then add a coronal one, rather than the other way round, resulting in the LC bias.

A second articulatory explanation known as the "LabialCoronal Chunking Hypothesis" was proposed by Sato, Vallee, Schwartz, and Rousset (2007). Their results from adult speeded articulation tasks showed that when French adults produce Consonant-Vowel-Consonant-Vowel sequences containing a labial (L) and a coronal (C) consonant at a fast articulatory rate, their productions tend to shift to Consonant-Consonant-Vowel sequences. When this happened, French adults produce LC-vowel sequences more frequently than CL-vowel sequences (e.g. both /bete/ and /tebe/ shifted to /b'te/). Based on these results (extended to Japanese adults by Tsuji, Gonzalez-Gomez, Medina, Nazzi, \& Mazuka, 2012), Sato, Vallee, Schwartz, and Rousset (2007) suggested that the LC bias might be explained by the higher articulatory stability of LC sequences compared with $\mathrm{CL}$ ones. It is important to emphasize that both articulatory explanations assume the existence of a language-general (that is, non-language specific) LC bias, predicting similar patterns across languages.

More recently, the importance of considering perceptual explanations for the LC bias has been stressed (Nazzi, Bertoncini, \& Bijeljac-Babic, 2009; GonzalezGomez \& Nazzi, 2012a). This perspective hypothesized that the LC bias results from infants' exposure to the linguistic input. This hypothesis was based on the previous observation of links existing between infants' preferences for specific sound sequences and their frequencies in the language. Indeed, prior studies have shown that by 9 months of age, infants have become sensitive to the phonotactic properties of their native language, preferring legal over illegal sequences (Jusczyk, Friederici, Wessels, Svenkerud, \& Jusczyk, 1993a; Friederici \& Wessels, 1993; Sebastián-Gallés \& Bosch, 2002), and also more frequent over less frequent phonotactically legal sequences (Jusczyk, Luce, \& Charles-Luce, 1994). With regard to the LC bias, it has been shown that LC sequences are significantly more frequent than $\mathrm{CL}$ sequences in the lexicons of 17 different languages (MacNeilage et al., 1999; Vallée et al., 2001). According to the perceptual-based perspective, the LC bias might thus be a result of infants' exposure to a linguistic input containing more $\mathrm{LC}$ than $\mathrm{CL}$ sequences.

The results of three recent perceptual studies seem to bring support to this perceptual hypothesis. The head-turn preference procedure (HPP) was used to explore Frenchlearning infants' preference for lists of LC or CL sequences (words or pseudo-words in French pronounced by a native female speaker). Results showed that between 7 and 10 months of age, French-learning infants start preferring the lists corresponding to the LC sequences, the significantly more frequent phonotactic structure in French (Nazzi et al., 2009; Gonzalez-Gomez \& Nazzi, 2012a,b). This was found even before infants start producing LC or CL structures, in both full term and preterm infants of the same chronological age, disfavoring the production interpretation; moreover, the data from the preterm infants further rules out the possibility of an emergence solely based on maturational level (Gonzalez-Gomez \& Nazzi, 2012a,b). 
Importantly, the predictions of the perceptual-based account change depending on the properties of the input: thus, if CL sequences are favored over LC sequences in the input, this account predicts a preference for CL over LC sequences. This contrasts with the above-mentioned articulatory explanations, which predict a general preference for LC patterns. In order to critically assess these perceptualbased predictions, Gonzalez-Gomez and Nazzi (2012c) conducted a more detailed analysis of the French lexicon, finding that the LC bias was not homogeneously present across consonantal classes in French: while the LC bias is clearly present for plosive (plosive consonants are produced by stopping airflow through the mouth, i.e., labials /p/ and / $\mathrm{b} / \mathrm{vs}$. coronals $/ \mathrm{t} /$ and $/ \mathrm{d} /$ ) and nasal sequences (nasals are produced by airflow through the nose, i.e., labial $/ \mathrm{m} / \mathrm{vs}$. coronal $/ \mathrm{n} /$ ), this is not the case for fricative sequences (fricatives are produced by forcing airflow through a narrow opening in the mouth i.e., labials /f/ and /v/ vs. coronals /s/ and $\mid z /$ ). Accordingly, Gonzalez-Gomez and Nazzi (2012c) tested the level of generalization at which these phonotactic acquisitions operate. In a series of experiments, 10month-old French-learning infants' preferences for LC or CL structures in plosive, nasal and fricative sequences were evaluated. The results indicated an LC preference for plosive and nasal sequences, but a $\mathrm{CL}$ preference for fricative sequences, suggesting that the LC bias reflects the properties of the input and is acquired at the level of classes of consonants defined by their manner of articulation.

The results of Nazzi, Bertoncini, and Bijeljac-Babic (2009) and Gonzalez-Gomez and Nazzi (2012a,b,c) thus seem to suggest that the early LC/CL biases are perceptually-based, reflect infants' learning about the structural regularities of the French lexicon, and result from exposure to the input rather than from maturation. Importantly, the results of Gonzalez-Gomez and Nazzi (2012c) on French further suggest that the perceptual LC bias (found for plosives and nasals) can be reversed in case the input shows a $\mathrm{CL}$ bias (for fricatives). Therefore, the contribution of Gonzalez-Gomez and Nazzi (2012c) is to establish that different types of consonants lead to different biases in the same infant population, as predicted by input frequencies in their native language. However, it cannot be completely excluded that for some yet-unidentified (linguistically-relevant or characteristic of the stimuli used) acoustic properties, plosives and nasals are processed differently than fricatives, leading to opposite LC/CL biases, independently of input frequencies ${ }^{1}$.

\footnotetext{
${ }^{1}$ Note also that the LC bias in early production was mainly based on sequences involving plosives or nasals, manner classes that are predominantly produced by young children (c.f., MacNeilage et al., 1999), so that the observed perceptual CL bias for fricative sequences in French-learning infants cannot be directly compared to those findings. Assuming that both perception and production play a role in shaping the bias, it could be the case that the input shapes a perceptual CL bias for fricatives in French, because early productions do not contain many fricative sequences. For plosive and/or nasal sequences, however, even input with a CL bias might not lead to a perceptual CL bias, because early productions contain a large amount of plosive and nasal LC sequences. It is therefore important to look for evidence for a perceptual CL bias for plosive and/or nasal sequences in a language where the input presents a CL bias, as found in Japanese.
}

Given this, another crucial step in showing the importance of early perceptual acquisition of input statistics in the emergence of $\mathrm{LC} / \mathrm{CL}$ biases is to show that the same stimuli are processed differently by infants learning languages that differ on this dimension at the input level. Therefore, as further motivated below, the present study used the same stimuli (plosive LC and CL words produced in Japanese) to investigate the emergence of different LC/ CL preferences in Japanese- and French-learning infants reflecting input differences in these two languages. This will also allow us to generalize to a language other than French the study of the emergence of LC/CL biases.

The choice for Japanese was originally motivated by the need to test a population learning a language having a lexicon that does not have a clear LC bias (contrary to French). According to MacNeilage et al. (1999) this is the case for Japanese. Their results showed not only that the Japanese lexicon does not have an LC bias, but that it tends to have the opposite pattern, that is a CL advantage. However, their results were based on a very small sample of words (68 words extracted from a travel dictionary), calling for more thorough analyses. Employing a larger database, Tsuji et al. (2012) found that the adult Japanese lexicon in fact has a general LC bias. However, a more fine-grained analysis based on the findings of GonzalezGomez and Nazzi (2012c) revealed that this bias is not homogenously distributed, but changes across consonant classes defined by manner of articulation: the overall LC bias extended to sequences of nasals, while a $\mathrm{CL}$ bias was found for plosive sequences.

Therefore, exploring the processing of plosive sequences in Japanese appeared as a good basis for further investigation of the perceptual factors determining the LC/CL biases. In this context, Tsuji et al. (2012) explored Japanese adults' production and perception of plosive sequences containing a labial consonant $(/ \mathrm{p} /$ or $/ \mathrm{b} /)$ and a coronal consonant $(/ \mathrm{t} /$ or $/ \mathrm{d} /)$. The results revealed that Japanese adults have an LC bias in production, supporting the explanations in terms of articulatory constraints (MacNeilage \& Davis, 2000a; MacNeilage et al., 2000; Sato et al., 2007). However, Japanese adults did show a perceptual CL bias for these plosive sequences, showing the influence of language exposure on perceptual biases as had been previously suggested (Nazzi et al., 2009; Gonzalez-Gomez \& Nazzi, 2012a,b,c). Based on these results, Tsuji et al. (2012) concluded that in adulthood there are two different biases, a productive LC bias due to constraints of the articulatory system, and a perceptual CL bias based on distributional frequencies in the lexicon. Moreover, these findings suggest that there might be a default LC bias, defined by articulatory constraints, which will partly (but not entirely) determine the structure of the lexicon across languages, and will lead to both production and perceptual LC biases. When exceptions to the LC bias are found in the input (for example, on fricative-fricative sequences in French, or on plosiveplosive sequences in Japanese), then a perceptual bias for the opposite CL bias is observed (perceptual CL bias for fricative sequences in French-learning infants, and plosive sequences in Japanese adults). The present study will explore whether a perceptual CL bias for plosive sequences can be found in Japanese-learning infants. 
The role that input properties play on the development of the perceptual LC bias was explored using exactly the same LC and CL sequences, recorded by a native Japanese speaker in two different infant populations. Given the properties of the Japanese and French lexicons, the input-based explanation will predict opposite preference patterns for plosive sequences: a CL preference for Japanese-learning infants and an LC preference for French-learning infants. Given previous data on French-learning infants, no biases should be found at 7 months of age, but language-specific biases should appear from 10 months onward (Nazzi et al., 2009; Gonzalez-Gomez \& Nazzi, 2012a,b,c).

Before conducting the perceptual studies, different frequency analyses were conducted in the Japanese lexicon, both in an infant-direct speech (IDS) corpus and in an adult-direct speech (ADS) corpus. This is important given that MacNeilage et al. (1999) used a corpus having a very small number of words, and that Tsuji et al. (2012) used only adult corpora. Thus the present analyses will first allow the verification of these phonotactic properties in the Japanese lexicon. Second, they will establish whether IDS shows a similar or a different pattern compared to ADS.

\section{Corpus study}

\subsection{Input}

IDS and ADS counts were obtained from the RIKEN Japanese Mother-Infant Conversation Corpus (R-JMICC, Mazuka, Igarashi, \& Nishikawa, 2006). First, IDS analyses were made in a corpus containing the conversations of 22 mothers with their 18-to-24-month-old infants in both toy-playing and book-reading environments (collapsed for the purpose of this analysis). The IDS corpus contains 42805 word tokens and 3482 word types. Second, the corpus includes a conversation of each mother with an experimenter on child-related topics but using adult-directed speech (ADS). The ADS corpus contains 22334 word tokens and 2523 word types. This corpus was analyzed separately.

\subsection{Analyses}

Given the differences in results for different manners of articulation in the French lexicon (Gonzalez-Gomez \& Nazzi, 2012c) and in Japanese ADS (Tsuji et al., 2012), we conducted one analysis including all consonant manners and three analyses restricted to sequences homogeneous in terms of manner of articulation: plosives, nasals, and fricatives. The overall analysis included 5 labials $/ \mathrm{p}, \mathrm{b}, \mathrm{m}$, $\phi, \beta /$ and 11 coronals $/ \mathrm{t}, \mathrm{d}, \mathrm{n}, \mathrm{s}, \mathrm{z}, \mathrm{c}, \widehat{\mathrm{ts}}, \widehat{\mathrm{t}_{\epsilon}}, \widehat{\mathrm{dz}}, \mathrm{j}, \mathrm{r} /$. The analysis of plosive sequences included labials /p, b/ and coronals $/ \mathrm{t}, \mathrm{d} /$; the analysis of nasal sequences included labial $/ \mathrm{m} /$ and coronal $/ \mathrm{n} /$; the analysis of fricative sequences contained labials $/ \phi, \beta /$ and coronals $/ \mathrm{s}, \mathrm{z}, c /$. Note that labial fricatives are very infrequent and, with the exception of / $\phi$ / preceding the vowel / $\mathrm{l} /$, appear exclusively in recent loanwords.

The majority of syllables in Japanese have a ConsonantVowel (CV) structure due to phonotactic constraints, and accordingly the majority of consonant-consonant dependencies occur in CVCV rather than CVC sequences. The only consonants that occur in coda position, and thus can form CVC sequences, are moraic nasals and geminate obstruents. We chose to exclude these from our analyses, because our experimental stimuli are CVCV sequences.

For each of the four types of sequences; four different frequency analyses were conducted, (1) token frequencies including CVCV sequences at any position within a word; (2) token frequencies for word-initial CVCV sequences only; (3) token frequencies of CVCV words; and (4) type frequencies of CVCV sequences at any position within a word. ${ }^{2}$

\section{Results and discussion}

Japanese ADS shows an overall LC bias, which is also found for nasal and fricative sequences; but it shows a strong CL bias for plosive sequences across counts (see Table 1). These ADS results obtained on a rather small corpus conform to the patterns found previously in an analysis of two larger corpora (Tsuji et al., 2012), thus supporting the representativeness of this smaller corpus.

Japanese IDS also shows an overall LC bias, and an LC bias for fricatives. Contrary to ADS, Japanese IDS exhibits a CL bias for sequences of nasals. However, caution is required in interpreting the data for fricatives and nasals since the number of tokens with fricative and nasal sequences in the corpus is rather small. Lastly, and again contrary to ADS, the plosive sequences also presented an overall LC bias. Importantly though, a CL bias was found for the analysis restricted to CVCV words, the structure of the stimuli used in Experiment 1.

The differences between ADS and IDS with regard to the subset of plosives are remarkable given the claims of a language-general preference for LC sequences in acquisition, which is mainly based on the production of plosives and nasals, and the reports of an LC bias across languages (MacNeilage et al., 1999). With regard to the only manner subset in Japanese ADS that goes against previously claimed language general tendencies (plosives), IDS markedly differs from ADS and follows the pattern that is more common across languages (except for CVCV words). By contrast, an analysis of the French lexicon showed that the LC bias is consistently present both in IDS and ADS, and both overall and for plosives, the pattern for nasal and fricative sequences being unspecified at this time given the very small number of tokens in our corpus (see Appendix A).

Taken together, the present findings indicate that, overall, Japanese is also an LC language, confirming the results found by Tsuji et al. (2012). These findings are consistent for the overall analysis (while caution is needed to interpret nasal and fricative patterns in the IDS corpus given again small numbers). However, plosive sequences showed a consistent CL bias in ADS, and in one of the four analyses in IDS (the CVCV words analysis, corresponding to the stimuli used in Experiments 1 and 2). Thus plosive sequences appear as relatively good candidates to test

\footnotetext{
2 Type frequencies for word-initial CVCV sequences only and for CVCV words were also explored, but are not reported here since the numbers were very low; However, they led to a pattern of results similar to the one found in the other reported analyses.
} 
Table 1

Absolute frequencies of LC and CL sequences and LC to CL ratios in the RJMIIC. Ratios above 1 indicate an LC bias, ratios below 1 indicate a CL bias (marked by grey shading).

\begin{tabular}{|c|c|c|c|c|c|c|c|c|}
\hline & \multicolumn{4}{|l|}{ IDS } & \multicolumn{4}{|l|}{ ADS } \\
\hline & Overall & Plosive & Nasal & Fricative & Overall & Plosive & Nasal & Fricative \\
\hline \multicolumn{9}{|c|}{ Token frequency } \\
\hline$\underline{\mathrm{LC}}$ & 1966 & 183 & 36 & 8 & 1028 & 31 & 79 & 8 \\
\hline$\overline{\mathrm{CL}}$ & 1297 & 142 & 47 & 2 & 823 & 155 & 31 & 1 \\
\hline$\overline{\text { Ratio }}$ & 1.52 & 1.29 & 0.77 & 4.00 & 1.25 & 0.20 & 2.55 & 8.00 \\
\hline \multicolumn{9}{|c|}{ Token frequency, word onset } \\
\hline$\underline{\mathrm{LC}}$ & 989 & 160 & 7 & 8 & 570 & 15 & 54 & 6 \\
\hline$\overline{\mathrm{CL}}$ & 773 & 136 & 39 & 0 & 499 & 122 & 24 & 1 \\
\hline Ratio & 1.28 & 1.18 & 0.18 & - & 1.14 & 0.12 & 2.25 & 6.00 \\
\hline \multicolumn{9}{|c|}{ Token frequency, CVCV words } \\
\hline$\underline{\mathrm{LC}}$ & $\overline{376}$ & 26 & 4 & 0 & 316 & 3 & 44 & 0 \\
\hline$\overline{\mathrm{CL}}$ & 347 & 96 & 17 & 0 & 266 & 61 & 9 & 0 \\
\hline Ratio & 1.08 & 0.27 & 0.24 & - & 1.19 & 0.05 & 4.89 & - \\
\hline \multicolumn{9}{|c|}{ Type frequency } \\
\hline$\underline{\mathrm{LC}}$ & 536 & 63 & 14 & 1 & 330 & 23 & 29 & 3 \\
\hline$\overline{\mathrm{CL}}$ & 360 & 24 & 19 & 2 & 277 & 28 & 17 & 1 \\
\hline$\overline{\text { Ratio }}$ & 1.49 & 2.63 & 0.74 & 0.50 & 1.19 & 0.82 & 1.71 & 3.00 \\
\hline
\end{tabular}

differential crosslinguistic perceptual biases in Japanese (CL bias, Experiment 1) and French (LC bias, Experiment 2). Accordingly, in Experiment 1, we tested the preferences of 7-, 10- and 13-month-old Japanese-learning infants for LC vs. CL plosive sequences. Three different possible outcomes were considered:

1. Emergence of a CL bias by 10 months. Given the results showing a perceptual CL bias in Japanese adults (Tsuji et al., 2012) and given the analyses of ADS, it is possible that Japanese-learning infants might develop a preference for CL sequences; based on previous studies with French-learning infants, this CL bias might emerge as early as 10 months of age.

2. Emergence of an LC bias by 10 months. Japanese-learning infants might show an LC bias if they are primarily focusing on IDS at this point of development. The Japanese IDS showed an overall LC bias for plosives (though a CL bias for CVCV words). If, like French-learning infants, Japanese infants begin to develop a preference for the pattern they hear frequently in their input, they may begin to show a preference for the LC pattern in plosives at about 10 months of age.

3. Emergence of a CL bias by 13 months. Given our contrasting findings between IDS and ADS for plosives, and since infants hear both IDS and ADS (van der Weijer, 2002; Soderstrom, 2007), it is possible that Japanese-learning infants might still show no clear preference at 10 months of age, and that a bias would emerge only at a later age, possibly by 13 months, when infants have accumulated enough evidence in support of a consistent CL bias in the lexicon (ADS).

\section{Experiment 1: Japanese-learning infants}

\subsection{Method}

\subsubsection{Participants}

Sixty infants from Japanese-speaking families were tested and their data included in the analyses: Twenty
Table 2

List of Labial-Coronal and Coronal-Labial $C_{1} V_{1} C_{2} V_{2}$ sequences used in the experiment.

\begin{tabular}{|c|c|c|c|}
\hline \multicolumn{2}{|c|}{ Labial-Coronal } & \multicolumn{2}{|c|}{ Coronal-Labial } \\
\hline Structure & Pseudo-word & Structure & Pseudo-worc \\
\hline & bado & & debi \\
\hline \multirow[t]{3}{*}{$b^{\mathbf{v}} \mathbf{d}$} & bida & $d^{\mathbf{v}} \mathbf{b}$ & dabe \\
\hline & bode & & dobe \\
\hline & peto & & tipa \\
\hline \multirow[t]{3}{*}{$p^{v} t$} & pita & $t^{v} \mathbf{p}$ & tipo \\
\hline & poti & & tope \\
\hline & beti & & tabo \\
\hline \multirow[t]{3}{*}{$b^{v} \mathbf{t}$} & beto & $t^{v} b$ & teba \\
\hline & bite & & tobi \\
\hline & pade & & depi \\
\hline \multirow[t]{2}{*}{$\mathbf{p}^{\mathbf{v}} \mathbf{d}$} & padi & $d^{\mathbf{v} p}$ & dipa \\
\hline & poda & & dapo \\
\hline
\end{tabular}

7-month-olds (mean age $=7$ months 13 days; range: 7 months 3 days - 24 days; 8 girls, 12 boys), twenty 10month-olds (mean age $=10$ months 8 days; range: 10 months 1 day - 29 days; 8 girls, 12 boys) and twenty 13-month-olds (mean age $=13$ months 11 days; range: 13 months 2 days - 25 days; 9 girls, 11 boys). The data of four additional 7-month-olds, four additional 10-montholds and 5 additional 13-month-olds were not included in the analyses due to fussiness/crying.

\subsubsection{Stimuli}

Twenty-four bisyllabic $\mathrm{C}_{1} \mathrm{~V}_{1} \mathrm{C}_{2} \mathrm{~V}_{2}$ pseudowords were selected (see Table 2), twelve items with a labial-coronal (LC) structure and twelve items with a coronal-labial (CL) structure. Items in both lists were made up of exactly the same consonants, and the vowels were almost completely balanced across lists. Vowels had been chosen in order to obtain balanced adjacent dependencies between the LC and $C L$ lists for the $C_{1} V_{1}, V_{1} C_{2}, C_{2} V_{2}$, and $C_{1} V_{1} C_{2} V_{2}$ sequences of phonemes according to R-JMIIC (Mazuka et al., 2006) and the NTT frequency corpus (Amano \& Kondō, 2000). It should be noted that both coronal and labial plosives in Japanese 
have restricted distributions. On the coronal side, $/ \mathrm{t} /$ and $/ \mathrm{d} /$ appeared only before /a/, /e/ and /o/ in traditional Japanese. Thus, although, /ti/ and / di/ are used regularly in loan words such as 'tii' (tea) and 'dinaa' (dinner) in modern Japanese, they occur relatively infrequently. ${ }^{3}$. On the labial side, /p/ occurs only in onomatopoeia, as an alternant of $/ \mathrm{h}, \mathrm{b} /$ in old (Yamato) Japanese, or in Western loanwords, making it the least frequent of all Japanese consonants (cf. Labrune, 2012, p. 60). Thus, although all CV sequences in the present study were legal, some of them occur less frequently than the others. The combination of the low frequency CV sequences together with the high frequency CV sequences allowed us to create $\mathrm{LC}$ and $\mathrm{CL}$ syllable sequences that are balanced in their adjacent dependencies.

The stimuli were recorded in a sound-attenuated booth by a Japanese female native speaker with the low-high pitch contour. Two tokens of each item were selected. Acoustic analyses showed that the stimuli were similar both in terms of duration $\left(\right.$ Mean $_{\text {total LC }}=438 \mathrm{~ms}$ vs. Mean $_{\text {total } \mathrm{CL}}=430 \mathrm{~ms}$; Mean $_{\text {syll. } 1 \mathrm{LC}}=163$ ms vs. Mean $_{\text {syll. } 1 \mathrm{CL}}=156 \mathrm{~ms} ;$ Mean $_{\text {syll. } 2 \mathrm{LC}}$ $=275 \mathrm{~ms}$ vs. Mean $\left._{\text {syll. } 1 \mathrm{CL}}=274 \mathrm{~ms}\right)$ and pitch $\left(\right.$ Mean $_{\text {total LC }}=$ $231 \mathrm{~Hz}$ vs. Mean total CL $=238 \mathrm{~Hz}$; Mean $_{\text {syll. } 1 \mathrm{LC}}=198 \mathrm{~Hz}$ vs. Mean $_{\text {syll.1 CL }}=203 \mathrm{~Hz}$; Mean syll.2 LC $=263 \mathrm{~Hz}$ vs. Mean syll. $1 \mathrm{CL}_{\mathrm{CL}}$ $=272 \mathrm{~Hz}$ ). Four lists were created: two lists with the twelve LC items (using different tokens across lists, the order of the items in the two lists being reversed) and two lists with the twelve CL items (same manipulation). The duration of all the lists was $18.0 \mathrm{~s}$.

\subsubsection{Procedure and apparatus}

The experiment was conducted inside a soundattenuated room, in a booth made of pegboard panels. The test booth had a red light and a loudspeaker mounted at eye level on each of the side panels and a green light mounted on the center panel. Below the center light was a video camera used to monitor infants' behavior.

A PC computer terminal, a camera, and a response box were located behind the center panel. The response box, connected to the computer, was equipped with a series of buttons. The observer, who looked at the video of the infant on the camera screen, pressed the buttons of the response box according to the direction of the infant's head, thus starting and stopping the flashing of the lights and the presentation of the sounds. The observer and the infant's caregiver listened to masking music over tightfitting closed headphones, which prevented them from hearing the stimuli presented. Information about the duration of the head-turn was stored on the computer.

The classic version of the Head-turn Preference Procedure (HPP) was used (Jusczyk, Cutler, \& Redanz, 1993b). Each infant was held on a caregiver's lap in the center of the test booth. Each trial began with the green light on the center panel blinking until the infant had oriented to it. Then, the red light on one of the side panels began to flash. When the infant turned in that direction, the stimulus for that trial began to play. The stimuli were delivered by the loudspeakers via an audio amplifier. Each stimulus

\footnotetext{
${ }^{3}$ These sequences were also used by mothers in IDS in the R-JMIIC corpus.
}

was played to completion or stopped immediately after the infant failed to maintain the head-turn for 2 consecutive seconds. If the infant turned away from the target by $30^{\circ}$ in any direction for less than 2 s and then turned back again, the trial continued but the time spent looking away was not included in the orientation time. Thus, the maximum orientation time for a given trial was the duration of the entire speech sample.

Each session began with two musical trials, one on each side to give infants an opportunity to practice one headturn to each side. The test phase consisted of two blocks (in each of which the two lists of each structure were presented). The order of the different lists within each block was randomized.

\subsection{Results and discussion}

Mean orientation times to the LC and CL lists were calculated for each infant. Orientation times lower than $1.5 \mathrm{~s}$ were excluded from the analysis (corresponding to 1 trial for two 7-month-olds, 1 trial for two 10-month-olds and 1 trial for six 13-month-olds) because the software used in France (Experiment 2) automatically rejects and replays such trials. Results were identical with or without these rejected trials.

The data for the Japanese-learning 7-month-olds $\left(M_{\mathrm{LC}}=8.72 \mathrm{~s}, \mathrm{SD}=2.36 \mathrm{~s} ; M_{\mathrm{CL}}=8.77 \mathrm{~s}, \mathrm{SD}=2.40 \mathrm{~s}\right), 10-$ month-olds $\left(M_{\mathrm{LC}}=10.14 \mathrm{~s}, \mathrm{SD}=3.58 \mathrm{~s} ; M_{\mathrm{CL}}=11.00 \mathrm{~s}\right.$, $\mathrm{SD}=3.23 \mathrm{~s})$ and 13 -month-olds $\left(M_{\mathrm{LC}}=7.35 \mathrm{~s}, \mathrm{SD}=2.93 \mathrm{~s}\right.$; $M_{\mathrm{CL}}=9.18 \mathrm{~s}, \mathrm{SD}=2.32 \mathrm{~s}$ ), were analyzed using a 2-way ANOVA with the between-subject factor of age ( 7 vs. 10 vs. 13 months) and the within-subject factor of lexical structure (LC vs. CL words). The main effect of lexical structure was significant, $F(1,57)=6.79, p=.01, \eta p^{2}=.11$, infants having longer orientation times to $\mathrm{CL}$ than to $\mathrm{LC}$ lists. In addition, the main effect of age was significant, $F(2,57)=4.73, p=.01, \eta p^{2}=.14$, due to differences in mean orientation times across age groups, which tended to be longer for the 10-month-olds. The interaction between age and lexical structure failed to reach significance $F(2,57)=6.79, p=.11$. However, given our a priori hypotheses for a lack of bias at 7 months, and a bias emerging at either 10 or 13 months, planned comparisons were conducted in each age group (see Fig. 1). They revealed that the lexical structure effect was not significant at either 7 months, $F(1,57)=.008, p=.92$, or 10 months, $F(1,57)=$ $1.88, p=.18$, but it was significant at 13 months, $F(1,57)=9.57, \quad p=.003, d=-.74$. Longer orientation times for CL stimuli were found in only 8 of the 20 7 -month-olds ( $p=.87$, binomial test) and 13 of the 20 10 -month-olds ( $p=.13$, binomial test), but in 16 of the 20 13-month-olds ( $p=.006$, binomial test). The comparisons further showed that the interaction between lexical structure and age restricted to the 7 - and 13-month-olds was significant, $F(1,57)=4.52, p=.04$, while that same interaction restricted to both the 7 - and 10-month-olds and to the 10- and 13-month-olds failed to reach significance $(F(1,57)=.82, p=.37$ and $F(1,57)=1.48, p=.23$, respectively).

Lastly, a Pearson's correlation was run to determine the relationship between age and the size of the lexical 


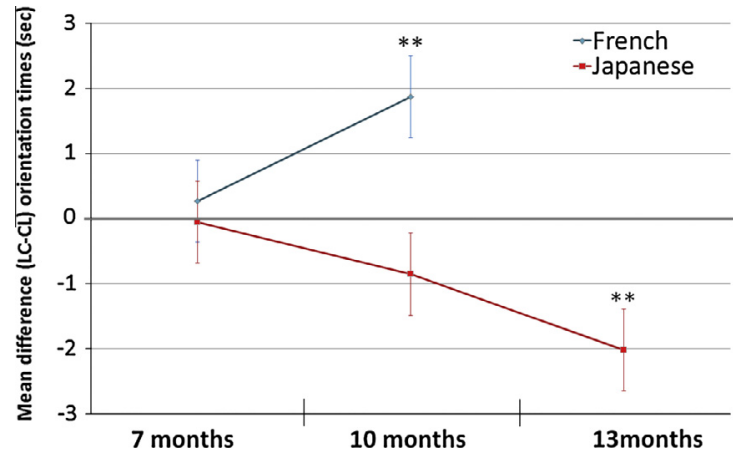

Fig. 1. Mean difference (LC-CL) orientation times (and SEs) for the 7-, 10-, and-13-month-old Japanese-learning infants in Exp. 1 and for the 7- and10-month-old French-learning infants in Exp. 2. Means above 0 indicate an LC bias, means below 0 indicate a CL bias.

structure effect (LC-CL). The results showed a significant negative correlation between age and size of the lexical structure effect $(r=-.27, N=60, p=.038)$, indicating that preference for $\mathrm{CL}$ over $\mathrm{LC}$ increased with age. The results of Experiment 1 establish the emergence of a perceptual $\mathrm{CL}$ bias between the ages of 7 and 13 months, with intermediate performance at 10 months of age.

Following the Japanese corpus analyses, we offered three possible predictions regarding Japanese-learning infants' preferences for LC/CL sequences. The CL preference found by 13 months of age and the lack of preference at both 7 and 10 months is compatible with the third possibility, according to which a CL preference emerges, but at a later age. This delay in the emergence of the CL preference is likely to be the result of the exposure to a linguistic input showing a weaker and less consistent CL bias than what has been found in the studies of French exploring the link between input and perceptual biases (Gonzalez Gomez \& Nazzi, 2012a,b,c). However, the present findings do not address the point we raised earlier, namely that the emergence of this CL preference, together with the delay in its emergence, might be due to idiosyncratic properties of the stimuli used, rather than to language-specific properties of the Japanese language. In order to exclude this idiosyncratic interpretation and support our preferred language-specific hypothesis, Experiment 2 was conducted using exactly the same stimuli and procedure, but this time testing a population exposed to a language showing a clear LC bias for plosive sequences in the lexicon, that is, French. Provided that infants can process the Japanese stimuli in a way similar to the way they have processed equivalent French stimuli in previous studies (Nazzi et al., 2009; Gonzalez-Gomez \& Nazzi, 2012a), we predict the emergence of an LC bias between 7 and 10 months of age.

\section{Experiment 2: French-learning infants}

\subsection{Method}

\subsubsection{Participants}

Forty infants from French-speaking families were tested and their data included in the analyses: Twenty 7-month-olds (mean age $=7$ months 9 days; range: 7 months 1 day - 23 days; 8 girls, 12 boys) and twenty 10 -month-olds (mean age $=10$ months 12 days; range: 10 months 1 day - 26 days; 11 girls, 9 boys). The data of two additional 7-month-olds and three additional 10 -month-olds were not included in the analyses due to fussiness/crying.

\subsubsection{Stimuli, procedure and apparatus}

They were the same as in Experiment 1, except for some minor apparatus differences. First, the PC computer terminal, a TV screen connected to the camera, and a response box were located outside the sound-attenuated room. Second, the observer looked at the video of the infant on the TV screen. Third, if a trial lasted less than 1.5s, the trial was automatically repeated and the original orientation time was discarded.

\subsection{Results and discussion}

Mean orientation times to the LC and CL lists were calculated for each infant. The data for the French-learning 7month-olds $\left(M_{\mathrm{LC}}=10.29 \mathrm{~s}, \mathrm{SD}=2.62 \mathrm{~s} ; M_{\mathrm{CL}}=10.01 \mathrm{~s}\right.$, $\mathrm{SD}=3.06 \mathrm{~s}$ ), and for the French-learning 10-month-olds $\left(M_{\mathrm{LC}}=8.83 \mathrm{~s}, \mathrm{SD}=2.39 \mathrm{~s} ; M_{\mathrm{CL}}=6.95 \mathrm{~s}, \mathrm{SD}=2.80 \mathrm{~s}\right)$ were analyzed using a 2-way ANOVA with the between-subject factor of age ( 7 vs. 10 months) and the within-subject factor of lexical structure (LC vs. CL words). The effect of lexical structure was significant, $F(1,38)=7.71, p=.008$, $\eta p^{2}=.17$, infants having longer orientation times to LC than to $\mathrm{CL}$ lists. The effect of age was significant, $F(1,38)=8.58, p=.006, \eta p^{2}=.18$, due to mean orientation time differences, which were longer for the 7-month-olds. Lastly, the interaction between age and lexical structure was significant, $F(1,38)=4.32, p=.04, \eta p^{2}=.10$, indicating that the effect of lexical structure changed with age (see Fig. 1). As for the Japanese data, and given our a priori predictions (no bias at 7 months, bias at 10 months), planned comparisons were conducted, showing that the effect of lexical structure was not significant at 7 months, $F(1,38)=.24, p=.63$, but was significant at 10 months, $F(1,38)=11.78, p=.002$. A bias for LC stimuli was found in only 11 of the 207 -month-olds ( $p=.41$, binomial test), but in 16 out of the 2010 -month-olds ( $p=.006$, binomial test). Lastly, as in Experiment 1, a Pearson's correlation was run to determine the relationship between age and the size of the lexical structure effect (LC-CL). The results revealed a positive correlation between age and size of the lexical structure effect $(r=.32, N=60, p=.044)$. These results confirm that an LC bias emerges between 7 and 10 months of age in French-learning infants, this preference being present even with stimuli recorded in a foreign language, here Japanese.

\section{General discussion}

The goal of the present study was to explore the role that linguistic input plays in the emergence of the LC bias. In the past, different studies have shown the emergence of an LC bias in early production studies (Ingram, 1974; 
MacNeilage et al., 1999; MacNeilage \& Davis, 2000b) and more recently at the perceptual level as well (Nazzi et al., 2009; Gonzalez-Gomez \& Nazzi, 2012a,b,c). Authors have attributed this bias on the one hand to language-general articulatory constraints on production favoring LC sequences (Ingram, 1974; MacNeilage et al., 2000; MacNeilage \& Davis, 2000a; Rochet-Capellan \& Schwartz, 2005; Sato et al., 2007), and on the other hand to language-specific effects of linguistic exposure on perceptual acquisition, resulting in LC or CL biases depending on the statistical properties of the input (Nazzi et al., 2009; Gonzalez-Gomez \& Nazzi, 2012a,b,c). However, all the acquisition studies on the perceptual bias had been conducted on a single linguistic population learning a language with a clear LC bias, French, and a CL bias was found in only one study on fricative sequences. Therefore, while the CL effects observed could indeed be related to properties of the native language, it was not possible to exclude the alternative interpretation that they could be due to idiosyncratic properties of the stimuli used, or to intrinsic yet undetermined properties of fricative sequences. Second, there was no comparative data showing the emergence of a CL bias previously found in adults, an important piece of data given that the articulatory and the perceptual account differ in their predictions only in cases in which the input favors a CL bias. In these cases, a CL bias is predicted for perception while an LC bias is predicted for production.

To address the hypothesis that the preference for LC or $\mathrm{CL}$ sequences at the perceptual level is determined by properties of the input of the native language, the present research explored the development of perceptual preferences for the same stimuli, plosive sequences containing a labial consonant and a coronal consonant, in Japaneseand French-learning infants. The goal was to test the input-based prediction that these two infant populations would process these stimuli differently. Our study revealed such crosslinguistic differences. For Japanese-learning infants, we found no evidence of an LC bias at any age. Instead, these infants showed a $\mathrm{CL}$ preference emerging between 7 and 13 months of age, with intermediate performance at 10 months (Exp. 1). In contrast, an LC preference emerging between 7 and 10 months was found in Frenchlearning infants, using the same stimuli recorded by a Japanese speaker (Exp. 2). These effects are predicted by the input properties in both languages, confirming that input exposure plays a crucial role in the emergence of such preferences, as previously argued (Nazzi et al., 2009; Gonzalez Gomez \& Nazzi, 2012a,b,c). Moreover, they demonstrate for the first time that these effects cannot be artifacts related to the (language-relevant or idiosyncratic) properties of the stimuli presented, since different effects are found crosslinguistically with the same stimuli.

Accordingly, the present results have implications for the interpretation of the LC bias. Classically, the effect has been explained as the result of production constraints (Ingram, 1974; MacNeilage \& Davis, 2000a; MacNeilage et al., 2000; Sato et al., 2007). In contrast, Nazzi et al. (2009) and Gonzalez-Gomez and Nazzi (2012a) offered a perceptual explanation. These two perspectives at first appear to be mutually exclusive. However, the results of
Tsuji et al. (2012) suggest that the LC bias has both a perceptual and an articulatory component. First, based on previous studies, we can conclude that there is a language-general LC bias in production (Ingram, 1974: Locke, 1983; MacNeilage \& Davis, 2000a; MacNeilage et al., 1999; Sato et al., 2007; Tsuji et al., 2012). Second, our results confirm the existence of language specific LC and $\mathrm{CL}$ biases. This means that not only does the emergence (or not) of the perceptual LC bias depends on exposure to a linguistic input showing such a bias, but exposure to a linguistic input showing an opposite tendency, that is a CL bias, triggers a perceptual preference for such sequences, as found for plosive sequences in Japaneselearning infants at 13 months of age (Exp. 1) and Japanese adults (Tsuji et al., 2012), and for fricative sequences in French-learning 10-month-olds (Gonzalez-Gomez \& Nazzi, 2012c). More importantly, the results of Tsuji et al. (2012) suggest that the two biases (articulatory and perceptual) can coexist in parallel: Japanese adults appear to have both an articulatory-based LC bias in production and an input-based CL bias in perception, which is influenced by distributional frequencies of the lexicon and emerges between 7 and 13 months of age. Based on these results, it is highly possible that during the 50 -word-stage, Japanese-learning infants would tend to produce more LC than CL structures even for plosive sequences, as has been previously observed in populations learning other languages (Ingram, 1974; Locke, 1983; MacNeilage et al., 1999; MacNeilage \& Davis, 2000a; see Vihman, 2014, for data on other phonotactic constraints), even if by 13 months of age Japanese-learning infants show a perceptual preference for the $\mathrm{CL}$ sequences. However, further studies are needed to explore this possibility.

Interestingly, our findings reveal a time-lag in the acquisition of the $\mathrm{LC} / \mathrm{CL}$ dependency between the Japanese-learning (Exp. 1) and French-learning (Exp. 2) infants. At present, several non-mutually exclusive factors could explain this developmental lag. First, remember that the frequency distribution of LC and CL plosive sequences in the Japanese lexicon was less clear than what has previously been observed in French: CL plosive sequences were more frequent in ADS input, while there was an advantage in favor of LC plosive sequences in IDS (except for CVCV structures). Thus, it might take longer for infants to accumulate enough evidence in support of the CL bias, because this bias is only present in ADS and in IDS CVCV structures. Second, a perceptual CL bias might be more difficult to learn than a perceptual LC bias, because it goes against what appears to be a language-general production LC bias (which will however need to be further evaluated in future studies), as is suggested for Japanese by the adults data obtained by Tsuji et al. (2012). These opposed production/perception biases might make the learning of these phonotactic dependencies more difficult, since several studies have shown the importance of the perception-production link (see Vihman, 1993; Vihman \& Croft, 2007; Yeung \& Werker, 2013) and the influence of production experience on infant speech processing (Keren-Portnoy, Vihman, DePaolis, Whitaker, \& Williams, 2010; DePaolis, Vihman, \& Keren-Portnoy, 2011; DePaolis, Vihman, \& Nakai, 2013; Majorano, Vihman, \& DePaolis, 2014). These 
different explanations are likely to be non-mutually exclusive, in particular in light of the fact that French-learning infants have been found to acquire a CL bias for fricative sequences without any developmental lag (e.g., by 10 months), a case that crucially differs from the present case in the fact that the input patterns were much clearer.

The mixed pattern of results found for ADS and IDS further raises important questions about the relative influence that IDS and ADS have on infants' speech perception during development. It is well known that by 4 months of age infants show a preference for IDS when compared to ADS (Cooper, Abraham, Berman, \& Staska, 1997; Cooper \& Aslin, 1990, 1994; Pegg, Werker, \& McLeod, 1992). However, it has also been shown that during the second half of the first year of life developmental changes take place in infant preferences for IDS (Newman \& Hussain, 2006; Hayashi, Tamekawa, \& Kiritani, 2001). Accordingly, it has been suggested that a decline in preference for IDS (Newman \& Hussain, 2006; Hayashi et al., 2001 ) and an increased role of ADS input on infants' language development (Soderstrom, 2007) takes place around 9 months of age. This period corresponds with the time when we begin to see changes in the LC/CL preference of Japanese-learning infants (10 months), opening the possibility that changes in the relative attention to IDS/ADS between 7 and 13 months contribute to the emergence of the CL preference. However further studies are needed to confirm this possibility.

Furthermore, while delayed emergence of a CL bias was found in Japanese, a different pattern of results was found for French-learning infants. The results of Experiment 2 showed that 10- but not 7-month-old French-learning infants have a preference for LC sequences, the structure that is more frequent in French, even when these sequences are produced in a foreign language (Japanese). These results are in line with studies, using French stimuli, showing the existence of a perceptual LC bias in 10-monthold French-learning infants, reflecting a preference for the typical phonotactic structures of French (Nazzi et al., 2009; Gonzalez-Gomez \& Nazzi, 2012a,b,c). Interestingly, the results of Experiment 2 indicate that French-learning infants' preference is not blocked by the acoustic/phonetic differences of the Japanese stimuli compared to native French phonology. These results contrast with those of Tsuji et al. (2012) showing that both Japanese and French adults were influenced by the language of the stimuli: Japanese adults showed a perceptual CL bias with the Japanese stimuli but not with the French ones, while French adults showed a perceptual LC bias only with the French stimuli. These effects had been interpreted as related to increased processing difficulty due to differences in acoustic/phonetic categories across the two languages (e.g., mostly unaspirated plosives in French, Fougeron \& Smith, 1993, vs. weakly aspirated plosives in Japanese, Okada, 1991). The fact that French-learning infants showed an LC bias both with French and Japanese stimuli suggests that at 10 months of age, infants still process Japanese phonemes as possible exemplars of French sounds, meaning that these sounds are still being assimilated as native.

To conclude, the present study, using the same stimuli consisting of plosive sequences, revealed the existence of crosslinguistic differences in the development of the

Table A1

Frequency ratios comparison of LC and CL sequences in French IDS (corpus by Karine Martel, Université de Caen Basse-Normandie) and ADS (Lexique 3 database; New, Pallier, Ferrand, \& Matos, 2001). Ratios above 1 indicate an LC bias, ratios below 1 indicate a CL bias (marked by grey shading).

\begin{tabular}{|c|c|c|c|c|c|c|c|c|}
\hline & \multicolumn{4}{|c|}{ IDS } & \multicolumn{4}{|c|}{ ADS } \\
\hline & Overall & Plosive & Nasal & Fricative & Overall & Plosive & Nasal & Fricative \\
\hline \multicolumn{9}{|c|}{ Token frequency } \\
\hline LC & 335 & 128 & 1 & 6 & 71822 & 9888 & 3566 & 6326 \\
\hline CL & 76 & 35 & 1 & 1 & 42772 & 5691 & 1063 & 6257 \\
\hline Ratio & 4.41 & 3.67 & 1.00 & 6.00 & 1.68 & 1.74 & 3.07 & 1.01 \\
\hline \multicolumn{9}{|c|}{ Token frequency, word onset } \\
\hline $\mathrm{LC}$ & 98 & 116 & 0 & 5 & 45323 & 6039 & 1648 & 3269 \\
\hline $\mathrm{CL}$ & 10 & 32 & 0 & 0 & 16144 & 4302 & 180 & 5240 \\
\hline Ratio & 9.80 & 3.63 & - & - & 2.81 & 1.40 & 9.18 & 0.62 \\
\hline \multicolumn{9}{|c|}{ Token frequency, CVCV words } \\
\hline $\mathrm{LC}$ & 98 & 25 & 0 & 5 & 6808 & 526 & 69 & 725 \\
\hline $\mathrm{CL}$ & 10 & 8 & 0 & 0 & 1178 & 295 & 0 & 329 \\
\hline Ratio & 9.80 & 3.13 & - & - & 5.77 & 1.78 & - & 2.20 \\
\hline \multicolumn{9}{|c|}{ Type frequency } \\
\hline $\mathrm{LC}$ & 109 & 15 & 1 & 3 & 13746 & 1853 & 1015 & 1331 \\
\hline CL & 88 & 8 & 0 & 1 & 8838 & 1269 & 412 & 784 \\
\hline Ratio & 2.86 & 1.88 & - & 3.00 & 1.56 & 1.46 & 2.46 & 1.70 \\
\hline
\end{tabular}


perceptual "LC bias", differences that were predicted by the properties of the lexicon of the languages contrasted (Japanese and French). Based on these results, it seems that exposure to linguistic input is a key factor in the emergence of the perceptual LC bias, establishing that an opposite CL bias can emerge if supported by the input. Taken together, these results suggest that in languages like Japanese, opposite biases can coexist in parallel (articulatory LC bias and perceptual CL bias), underlying the contributions of both perceptual and production factors on infants' phonological development. Furthermore, these results contribute to the understanding of some of the mechanisms underlying early language processing.

\section{Acknowledgments}

This study was conducted with the support of a CONACYT grant and a RIKEN summer internship grant to NGG, and an ANR-ESRC grant \# ANR-09-FRBR-015 to TN. We thank Yuri Hatano, Mihoko Hasegawa and Chiemi Oshino for their invaluable help in technical set-up stimuli recording and recruitment. Special thanks to the infants and their parents for their kindness and cooperation.

\section{Appendix A}

See Table A1.

\section{References}

Amano, S., \& Kondō, T. (2000). Nihongo no goitokusei (Lexical properties of Japanese) (Vol. 7), Frequency, NTT database series. Tokyo: Sanseidō.

Cooper, R. P., Abraham, J., Berman, S., \& Staska, M. (1997). The development of infants' preference for motherese. Infant Behavior and Development, 20, 477-488.

Cooper, R. P., \& Aslin, R. N. (1990). Preference for infant-directed speech in the first month after birth. Child Development, 61, 1584-1595.

Cooper, R. P., \& Aslin, R. N. (1994). Developmental differences in infant attention to the spectral properties of infant-directed speech. Child Development, 65, 1663-1677.

Davis, B. L., \& MacNeilage, P. F. (2004). The frame/content theory of speech evolution: From lip smacks to syllables. Primatologie, 6, 305-328.

DePaolis, R. A., Vihman, M. M., \& Keren-Portnoy, T. (2011). Do production patterns influence the processing of speech in prelinguistic infants? Infant Behavior and Development, 34, 590-601.

DePaolis, R. A., Vihman, M. M., \& Nakai, S. (2013). The influence of babbling patterns on the processing of speech. Infant Behavior and Development, 36, 642-649.

Fougeron, C., \& Smith, C. L. (1993). French. Journal of the International Phonetic Association, 23, 73-76.

Friederici, A. D., \& Wessels, J. M. I. (1993). Phonotactic knowledge and its use in infant speech perception. Perception \& Psychophysics, 54, 287-295.

Gonzalez-Gomez, N., \& Nazzi, T. (2012a). Acquisition of non-adjacent phonological dependencies in the native language during the first year of life. Infancy, 17, 498-524.

Gonzalez-Gomez, N., \& Nazzi, T. (2012b). Phonotactic acquisition in healthy preterm infants. Developmental Science, 15, 885-894.

Gonzalez-Gomez, N., \& Nazzi, T. (2012c). Phonological feature constraints on the acquisition of phonological dependencies. BUCLD Proceedings, 36, 202-2012.

Hayashi, A., Tamekawa, Y., \& Kiritani, S. (2001). Developmental change in auditory preference for speech stimuli in Japanese infants. Journal of Speech and Hearing Disorders, 44, 1189-1200.

Ingram, D. (1974). Fronting in child phonology. Journal of Child Language, $1,233-241$.
Jusczyk, P. W., Cutler, A., \& Redanz, N. (1993b). Preference for the predominant stress patterns of English words. Child Development, 64, 675-687.

Jusczyk, P., Friederici, A., Wessels, J., Svenkerud, V., \& Jusczyk, A. M. (1993a). Infants' sensibility to the sound patterns of native language words. Journal of Memory and Language, 32, 402-420.

Jusczyk, P. W., Luce, P. A., \& Charles-Luce, J. (1994). Infants' sensibility to phonotactic patterns in the native language. Journal of Memory and Language, 33, 630-645.

Kawasaki-Fukumori, H. (1992). An acoustical basis for universal phonotactic constraints. Language and Speech, 35, 73-86.

Keren-Portnoy, T., Vihman, M. M., DePaolis, R. A., Whitaker, C. J., \& Williams, N. M. A. (2010). The role of vocal practice in constructing phonological working memory. Journal of Speech, Language and Hearing Research, 53, 1280-1293.

Labrune, L. (2012). The phonology of Japanese. New York: Oxford University Press.

Locke, J. L. (1983). Phonological acquisition and change. New York: Academic Press.

MacNeilage, P. (1998). The frame/content theory of evolution of speech production. Behavioral and Brain Sciences, 21, 499-511.

MacNeilage, P. F., \& Davis, B. L. (2000b). On the origin of the internal structure of word forms. Science, 288(5465), 527-531.

MacNeilage, P. F., \& Davis, B. L. (1990). Acquisition of speech production: Achievement of segmental independence. In W. I. Hardcastle \& A. Marchal (Eds.), Speech production and speech modeling (pp. 55-68). Dordrecht: Kluwer.

MacNeilage, P. F., \& Davis, B. L. (2000a). The evolution of speech: The relation between ontogeny and phylogeny. In J. R. Hurford, C. Knight, \& M. G. Studdert-Kennedy (Eds.), The evolutionary emergence of language. Cambridge: Cambridge University Press.

MacNeilage, P. F., Davis, B. L., Kinney, A., \& Matyear, C. L. (1999). Origin of serial-output complexity in speech. Psychological Science, 10, 459-460.

MacNeilage, P. F., Davis, B. L., Kinney, A., \& Matyear, C. L. (2000) The motor core of speech: A comparison of serial organization patterns in infants and languages. Child Development, 71 153-163.

Majorano, M., Vihman, M. M., \& DePaolis, R. A. (2014). The relationship between infants' production experience and their processing of speech. Language Learning and Development, 10, 179-204.

Mazuka, R., Igarashi, Y., \& Nishikawa, K. (2006). Input for learning Japanese: RIKEN Japanese mother-infant conversation corpus. The Institute of Electronics, Information and Communication Engineers Technical Report, 16, 11-15.

Nazzi, T., Bertoncini, J., \& Bijeljac-Babic, R. (2009). Early emergence of a perceptual LC bias. Journal of the Acoustical Society of America, 126, 1440-1446.

New, B., Pallier, C., Ferrand, L., \& Matos, R. (2001). Une base de données lexicales du français contemporain sur internet: LEXIQUE. L'Année Psychologique, 101, 447-462.

Newman, R. S., \& Hussain, I. (2006). Changes in preference for infantdirected speech in low and moderate noise by 5 - and 13 -month-olds. Infancy, 10, 61-76.

Okada, H. (1991). Japanese. Journal of the International Phonetic Association, 21, 94-96.

Pegg, J. E., Werker, J. F., \& McLeod, P. J. (1992). Preference for infantdirected over adult-directed speech: Evidence from 7-week-old infants. Infant Behavior and Development, 15, 325-345.

Rochet-Capellan, A., \& Schwartz, J. (2005). Contraintes motrices et effet Labial-Coronal: Stabilité de bisyllabes $L C$ et $C L$ répétés de manière accélérée. Toulouse: Dans Actes des Rencontres Jeunes Chercheurs en Parole.

Rousset, I. (2003). From lexical to syllabic organization. In Proceedings of the XVth international congress of phonetic sciences ICPhS (pp. 715718), Barcelona.

Sato, M., Vallee, N., Schwartz, J. L., \& Rousset, I. (2007). A perceptual correlate of the labial-coronal effect. Journal of Speech Language and Hearing Research, 50, 1466-1480.

Sebastián-Gallés, N., \& Bosch, L. (2002). Building phonotactic knowledge in bilinguals: Role of early exposure. Journal of Experimental Psychology: Human Perception and Performance, 28, 974-989.

Soderstrom, M. (2007). Beyond babytalk: Re-evaluating the nature and content of speech input to preverbal infants. Developmental Review, 27, 501-532.

Tsuji, S., Gonzalez-Gomez, N., Medina, V., Nazzi, T., \& Mazuka, R. (2012). The labial-coronal effect revisited: Japanese adults say pata, but hear tapa. Cognition, 125, 413-428. 
Vallée, N., Rousset, I., \& Boë, L. J. (2001). Des lexiques aux syllabes des langues du monde. Typologies, tendances et organisations structurelles. Linx, 45, 37-50.

Van der Weijer, J. (2002). How much does an infant hear in a day? Paper presented at the Gala 2001 conference on language acquisition. Portugal: Lisboa.

Vihman, M. M. (1993). Variable paths to early word production. Journal of Phonetics, 21, 61-82.
Vihman, M. M. (2014). Phonological development: The first two years (2nd ed.). Malden, MA: Wiley-Blackwell.

Vihman, M. M., \& Croft, W. (2007). Phonological development: Toward a 'radical' templatic phonology. Linguistics, 45, 683-725.

Yeung, H. H., \& Werker, J. F. (2013). Lip movements affect infant audiovisual speech perception. Psychological Science, 24, 603-612. 